\title{
An ischemic stroke effectively treated with systemic thrombolytic therapy in an underage patient: clinic and ethics
}

\author{
Federica Stella***, Erica Pistollato*/** , Foscarina della Rocca*, Gianna Vettore*, Franco Tosato* \\ * Dipartimento di Emergenza ed Accettazione, Azienda Ospedaliera di Padova \\ ** Università degli Studi di Padova
}

\begin{abstract}
We describe the clinical case of a 15-year-old boy presenting with acute onset of left hemiplegia due to ischemic stroke, successfully treated with systemic thrombolysis with t-PA.

Diagnosis and management of paediatric stroke can be difficult in the Emergency Departement (ED), because of the rarity of the disease and because of the absence of clear guidelines guaranteeing a uniform treatment.
\end{abstract}

\section{Case report}

A 15-year-old boy was admitted to our ED because of an acute onset of weakness on his left hemisoma.

At 3 PM the boy was at home in the bathroom, during Valsalva manoeuvre he had felt a sudden weakness in his left arm and leg. He fell to the ground hitting his left shoulder and hip against the bath. His relatives immediately called the Emergency Medical Services (EMS) to take the boy to the ED, there he received a yellow code because of the focal neurological deficiency which had appeared less than 3 hours previously.

His medical history was unremarkable except for migraine without aura when he was a child; he had not been under under any medication, he denied any drug intake and no allergies to medicine were reported.

At ED admission, at 4.00 PM, the vital signs were the following: AVPU: Alert; awake, responsive, inspective ABC unremarkable, GCS = 15 (M6, V5, E4), HR 83 bpm, rhythmic; BP 130/60 mmHg; respiratory rate 14/min, $\mathrm{spO}_{2}=100 \%$ with no oxygen supplementation; body temperature $36.2^{\circ} \mathrm{C}$; serum glucose $94 \mathrm{mg} / \mathrm{dl}$. 12-lead EKG, acid-base balance and blood gases were normal. Serum basic chemistry was unremarkable, in particular no clotting disorders were revealed. A standard drug test was performed and resulted negative.

The neurological examination revealed: pupils bilaterally isochoric, isocyclic and reactive to light and accomodation, central facial hemiparesis on his left side, the other cranial nerves were grossly intact; left hemiplegia with flaccid hypotonus and left hemisoma anaesthesia, positive left Babinski's sign, normal and symmetric deep tendon reflexes, no dysphasia, no cerebellar and visual deficits, no neglect, NIHSS $=18$. Cardio-pulmunary and abdominal examination was unremarkable. No carotideal bruit was revealed.

Direct CT scan, performed at 4.30 PM, revealed hyperdensity in the horizontal tract of the right middle cerebral artery (MCA) and in its main branches, no other abnormalities (Fig. 1).

Carotid Doppler Ultrasonography revealed right internal carotid artery occlusion. Transcranial Doppler Ultrasonography revealed right MCA and ACA occlusion.

The neurologist confirmed our clinical suspect of ischemic stroke and considered the patient eligible for thrombolytic systemic therapy.

Given the minor age of the patient, both parents were in agreeance in the decision regarding the opportunity of performing thrombolytic therapy. Both were clearly informed of the risks and of the absence of clear guidelines for underage patient therapy; both of them agreed to the thrombolysis. The Hospital Administration and the regional Ethic Committee were contacted and, considering the severity of the clinical presentation and the fatal prognosis, even though underage patients are excluded from thrombolytic therapy, the parents agreed to their son receiving the emergency administration of tPA.

At 5.20 PM systemic thrombolytic therapy with recombinant tissue-type plasminogen activator (tPA; Alteplase TM) at dosage $0.9 \mathrm{mg} / \mathrm{kg}$ was started, an initial bolus of $7 \mathrm{mg}$ followed by continous infusion of $56 \mathrm{mg}$, concluded at $6.20 \mathrm{PM}$. At infusion end there was a NIHSS improvement from 18 to 14. No early thrombolytic complications were detected, a second CT scan at 7 PM revealed no haemorragies and revealed an attenuation of the hyperdensity of the right middle cerebral artery.

The patient was therefore transferred to the Neurological Stroke Unit to undergo further exams and treatment.

Diffusion-weighted imaging RMI (DWI-RMI) performed during the second day of hospitalization detected a wide ischemic lesion in the right MCA territory with recanalization of the right MCA; no images referable to carotid dissection.

Transcranial Doppler ultrasonography with bubble test revealed a left-to-right heart shunt with detection of numerous (> 10) microembolic signals (MES) at rest and "shower effect" ( $>25)$ during Valsalva Manoeuvre. Transthoracic echocardiogram was performed and confirmed the patency of the foramen ovale (PFO) of $2 \mathrm{~mm}^{2}$ with left-to-right shunt, no other structural or functional cardiac abnormalities were detected. 


\section{clinica e terapia}

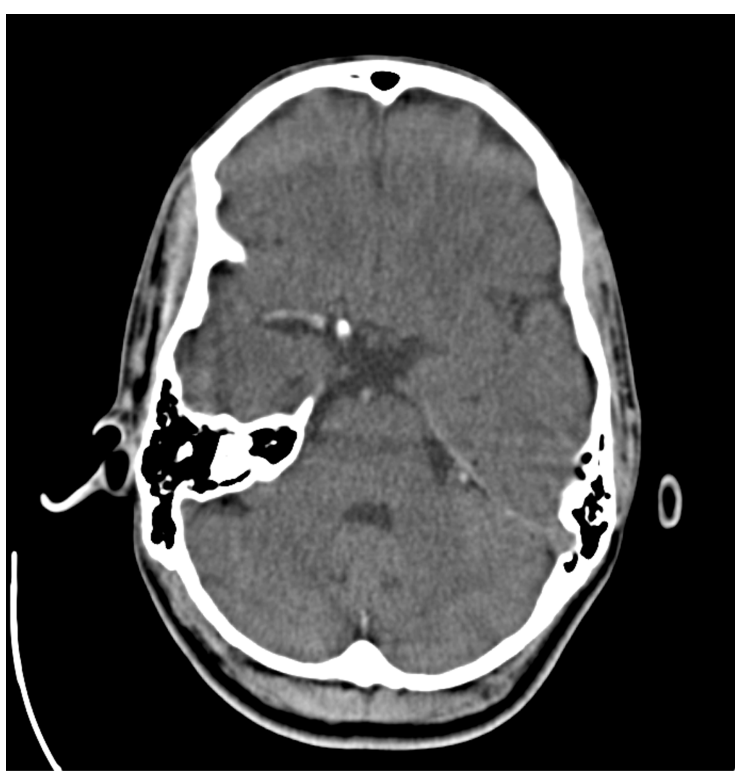

Fig. 1.

Several inherited prothrombotic risk factors were examinated, in particular the ones that are most commonly associated to stroke in the peadiatric population (1), such as factor V, factor II, and MTHFR mutations, and antiphospholipid antibodies; the performed panel was entirely normal.

Vital parameters were stable during hospitalization. Daily physiotherapy was performed obtaining good motor recovery with a decrease in NIHSS from 14 to 3 by the $7^{\circ}$ day and Barthel index rose from $10 / 100$ (value at time of admission) to $85 / 100$ (value at time of discharge), demonstrating an appreciable gradual improvement of residual authonomy.

At dismission the patient presented a mild motor deficiency at the left lower limb with a moderate left grab deficiency, normal Mingazzini I, unstable Mingazzini II, NIHSS 2.

The patient was discharged after 19 days with a diagnosis of "right MCA ischemic stroke with PFO with left-to-right shunt" and transferred to a rehabilitation care centre.

\section{Discussion}

Haemorrhagic and ischemic stroke is a rare disease in the paediatric population, yet there has been a recognised increase in recent years.

Diagnosis and management can be difficult due to the diversity of underlying risk factors and to the absence of guidelines guaranteeing a uniform treatment.

The WHO (World Health Organization) definition of stroke is "a clinical syndrome of rapidly developing focal or global disturbance of brain function lasting more than 24 hours". This definition is not congenial in children, because of shaded neurological symptoms or of overlapping clinical presentations of several clinical syndromes. Moreover, children and adolescents with stroke have notable differences in presentation compared to adults. Children with symptoms compatible with a transient ischemic attack (TIA) could have a brain infarction revealed by brain imaging despite the transient nature of their symptoms. Children with cerebral venous sinus thrombosis can commonly present headache or seizures at onset. "Stroke-like episodes" without a clear vascular cause may occur in migraine or metabolic disease (2).

Stroke type in paediatric patients also varies considerably. In Western countries, $80 \%$ to $85 \%$ of strokes among adults are ischemic, $15-20 \%$ are haemorrhagic. In children, more than $55 \%$ of strokes are ischemic, $45 \%$ are haemorrhagic.

The estimated annual stroke incidence rate for children in the main literature reviews is 2.1-2.7 per 100000 per year for strokes, even if the frequency of ischemic stroke appears to be greater than previously suggested (3).

In the underage population, peak age for ischemic stroke is in the first year of life, representing a third of the cases.

Although there have been a few reports of the use of tissue plasminogen activator (tPA) in children with ischemic stroke (46), safety and efficacy data for either intravenous or intra-arterial thrombolysis in children with acute arterial occlusion are lacking.

As in adults receiving tPA, it is likely that its delayed administration in children will lead to an unacceptable rate of intracerebral haemorrhage. If tPA is considered, it is imperative to adhere to the accepted time limits used in adults: administration of intravenous tPA within 3 hours of stroke onset and intra-arterial tPA within 6 hours of stroke onset for anterior circulation strokes. This is a serious limitation in children with stroke because the awareness of the severity and consequent rapidness in seeking help is much later than in older patients with stroke.

Even tPA dosage is subject to discussion, there is some evidence that a lower-dose tPA regimen might well be effective in children, with a remarkable complications reduction (7). If this observation is valid, it could be the startpoint for additional trials designed to improve safety usage of tPA in underage patients.

\section{Conclusions}

The treatment of stroke in infants and children is both important and understudied. Sometimes the treatment decisions are relatively clear, but more often the lack of randomized controlled clinical trials makes it difficult to know the clinical action to take.

Given the lack of clear guidelines, every single clinical case should be considered singularly.

If the clinical suspect is sustained by laboratory and instrumental findings and if the clinical onset is severe, considering that a conventional therapy could lead to high morbidity and residual disability, administration of tPA for a systemic thrombolysis should be at least considered. 
To achieve a correct management of these difficult clinical cases and to obtain an off-label administration of a drug approved from EMEA for different patients, it must be possible for the Emergency Physician to get in touch with the Hospital Administration and regional Ethic Committee. When the go-ahead is obtained, clear explanation of potential benefits and risk of the procedure should be explained to the patients and to the parents or legal guardians of the child. After which, both the parents should give their informed consent and the child should give his/her informed assent, and an informed consent form should be signed.

Our clinical experience achieved excellent results, with a complete return to normal neurological function and no residual loss of function. This should point us towards considering an off-label therapy.

\section{References}

1. Kenet G, Sadetzki S, Murad H, Martinowitz U, Rosenberg N, Gitel S, Rechavi G, Inbal A. Factor V Leiden and antiphospholipid antibodies are significant risk factors for ischemic stroke in children. Stroke 2000; 31(6): 1283-88.

2. Roach ES, Golomb MR, Adams R, Biller J, Daniels S, deVeber G, Ferriero D, Jones BV, Kirkam FJ, Scott RM, Smith ER. Management of stroke in infants and children: a scientific statement from a special writing group of the American Heart Association Stroke Council and the Council on Cardiovascular Disease in the Young. Stroke 2008; 39: $2644-91$.

3. Lynch JK, Hitz DG, De Veber, Nelson KB. Report of the National Institute of Neurological Disorders and Stroke Workshop on perinatal and childhood stroke. Paediatrics 2002; 109: 116-123.

4. Carlson MD, Leber S, Deveikis J, Silvestein FS. Successful use of rt-PA in pediatric stroke. Neurology 2001; 57: 157-8.

5. Thirumalai SS, Shubin RA. Successful treatement for stroke in a child using recombinant tissue plasminogen activator. J Child Neurol 2000; 15: 558.

6. Janjua N, Nasar A, Lynch JK, Qureshi AI. Thrombolysis for ischemic stroke in children: data from the nationwide inpatient sample. Stroke 2007; 38: 1850-54.

7. Wang M, Hays T, Balasa V, Bagatell R, Gruppo R, Grabowski EF, Valentino LA, Tsao-Wu G, Manco-Johnson MJ. Pediatric Coagulation Consortium. Low-dose tissue plasminogen activator thrombolysis in children. J Pediatr Hematol Oncol 2003; 29: 415-24. 